\title{
Temporal genetic differentiation in Glossina pallidipes tsetse fly populations in Kenya
}

Winnie A. Okeyo ${ }^{1,2,3^{*}+}$, Norah P. Saarman ${ }^{4^{*}+}$, Michael Mengual ${ }^{4}$, Kirstin Dion ${ }^{4}$, Rosemary Bateta ${ }^{2}$, Paul O. Mireji ${ }^{1,2,5}$, Sylvance Okoth², Johnson O. Ouma ${ }^{2,6}$, Collins Ouma ${ }^{3}$, Joel Ochieng ${ }^{7}$, Grace Murilla², Serap Aksoy ${ }^{1}$ and Adalgisa Caccone $e^{1,4}$

\begin{abstract}
Background: Glossina pallidipes is a major vector of both Human and Animal African Trypanosomiasis (HAT and AAT) in Kenya. The disease imposes economic burden on endemic regions in Kenya, including south-western Kenya, which has undergone intense but unsuccessful tsetse fly control measures. We genotyped $387 \mathrm{G}$. pallidipes flies at 13 microsatellite markers to evaluate levels of temporal genetic variation in two regions that have been subjected to intensive eradication campaigns from the 1960s to the 1980s. One of the regions, Nguruman Escarpment, has been subject to habitat alteration due to human activities, while the other, Ruma National Park, has not. In addition, Nguruman Escarpment is impacted by the movement of grazing animals into the area from neighboring regions during the drought season. We collected our samples from three geographically close sampling sites for each of the two regions. Samples were collected between the years 2003 and 2015, spanning $\sim 96$ tsetse fly generations.

Results: We established that allelic richness averaged 3.49 and 3.63, and temporal $\mathrm{N}_{\mathrm{e}}$ estimates averaged 594 in Nguruman Escarpment and 1120 in Ruma National Park. This suggests that genetic diversity is similar to what was found in previous studies of G. pallidipes in Uganda and Kenya, implying that we could not detect a reduction in genetic diversity following the extensive control efforts during the 1960s to the 1980s. However, we did find differences in temporal patterns of genetic variation between the two regions, indicated by clustering analysis, pairwise $\mathrm{F}_{\mathrm{ST}}$, and Fisher's exact tests for changes in allele and genotype frequencies. In Nguruman Escarpment, findings indicated differentiation among samples collected in different years, and evidence of local genetic bottlenecks in two locations previous to 2003, and between 2009 and 2015. In contrast, there was no consistent evidence of differentiation among samples collected in different years, and no evidence of local genetic bottlenecks in Ruma National Park.

(Continued on next page)
\end{abstract}

\footnotetext{
*Correspondence: winnie.okeyo@yale.edu; norah.saarman@yale.edu

†Equal contributors

${ }^{1}$ Yale School of Public Health, Yale University, New Haven, CT, USA

${ }^{4}$ Department of Ecology \& Evolutionary Biology, Yale University, New Haven,

$C T$, USA

Full list of author information is available at the end of the article
} 
(Continued from previous page)

Conclusion: Our findings suggest that, despite extensive control measures especially between the 1960s and the 1980s, tsetse flies in these regions persist with levels of genetic diversity similar to that found in populations that did not experience extensive control measures. Our findings also indicate temporal genetic differentiation in Nguruman Escarpment detected at a scale of $>80$ generations, and no similar temporal differentiation in Ruma National Park. The different level of temporal differentiation between the two regions indicates that genetic drift is stronger in Nugruman Escarpment, for as-yet unknown reasons, which may include differences in land management. This suggests land management may have an impact on G. pallidipes population genetics, and reinforces the importance of long term monitoring of vector populations in estimates of parameters needed to model and plan effective species-specific control measures.

Keywords: Effective population size, Glossina pallidipes, Microsatellites, Population bottleneck, Temporal changes in allelic frequencies, Vector control

\section{Background}

Tsetse flies (genus Glossina) are the major insect vectors of Human African Trypanosomiasis (HAT) and Animal African Trypanosomiasis (AAT), serious animal and human diseases in sub-Saharan Africa [1-3]. HAT causes fatalities of thousands of people every year, while AAT, also known as Nagana, causes significant mortality in livestock with major economic losses impeding agricultural development [1-3]. Direct prevention and treatment of the diseases has not been possible because no vaccines exist against $\mathrm{HAT}$ and AAT, and available drugs for treatment are difficult to administer $[1,4]$ and drug resistance has been reported $[5,6]$. In view of the above, vector control therefore constitutes a major cornerstone of HAT and AAT suppression in Africa. Tsetse control methods include habitat interference, trapping, use of insecticide-treated targets, aerial or ground spraying, insecticide-treated cattle, or the release of sterile/transgenic insects [4, 7-10]. Despite implementation of vector control methods, their success has been limited by the required long timescales and large geographical scales necessary to control tsetse populations. Vector control methods also depend on complex coordination to maximize both their efficiency and long-term monitoring efforts [11].

In Kenya, HAT was first reported in 1902, and expanded during the mid-1950s until control efforts between the1960s and 1980s led to eradication of HAT from most of Kenya [11]. HAT is now limited to a small part of western Kenya along the Ugandan border [11], but re-emergence into regions such as southwestern Kenya remains a critical threat [11-13]. Currently, however, it is AAT that causes the largest threat to human wellbeing through the loss of income, when livestock become sick or die of the disease [14]. The major vector of AAT in Kenya is Glossina pallidipes, which occurs in savannah, shrub lands, and grassy woodland habitats that match specific conditions of humidity, rainfall and temperature $[15,16]$. Suitable conditions occur in non- contiguous patches that are concentrated along the southwestern and coastal borders of Kenya [14], and are at peak suitability during wet seasons [16]. The discontinuous and fluctuating nature of G. pallidipes habitat [20] has the potential to cause rapid changes in distribution and population density, and makes the direct monitoring of G. pallidipes difficult [15]. One tool that has been helpful in understanding underlying mechanisms of tsetse resurgence after control has been population genetics analyses [4, 17, 18]. Population genetics can thus contribute greatly to our understanding of best practices in vector control and monitoring $[17,18]$.

To date, population genetics studies of G. pallidipes in Kenya have shown high levels of genetic differentiation among micro- and macrogeographical scales [14, 19], but no significant changes in allelic frequencies across short time scales [16]. Previous work found five strong genetic breaks at a macro-geographical scale of hundreds of kilometers [14], and variable but minor differentiation at a micro-geographical scale of $10-30 \mathrm{~km}$ [19]. Differentiation across temporal scales has been less well studied. Work by Ouma et al. [16] in southwestern Kenya found no significant changes in allelic frequencies among collections temporally separated by 312 months ( 2-8 generations) and found that there were signals of recent population bottlenecks [16]. These results suggest there have not been large shifts in allele frequencies in G. pallidipes, at least not over short time periods. In contrast, work by Ciosi et al. [20] demonstrated shifts in allele frequencies in populations along the Uganda/Kenya border in samples collected in 2000 that they attributed to a bottleneck resulting from control measures in the 1990s. Thus, temporal shifts in allele frequencies appear to be context dependent, and there is a need for further research to distinguish potential drivers of genetic changes in G. pallidipes.

In this study, we use 13 microsatellite loci to describe temporal patterns of genetic variation in G. pallidipes 
populations across longer time scales than previously reported. We focus on two regions of southwestern Kenya, Nguruman Escarpment (Nguruman) and Ruma National Park (Ruma), that share similarities in ecology and evolutionary processes, and a history of successful suppression in the 1960s to the 1980s [21-23] with recent resurgence in tsetse fly numbers $[14,16,19,24,25]$. However, these regions differ in land management. Nguruman does not restrict human use and allows grazing, agriculture and fire wood collection [26-29], whereas Ruma is a National Park that strictly prohibits all of these human activities in the interest of wildlife conservation [30]. Here, we evaluate and compare temporal patterns of genetic variation in these two regions, making use of previous collections made in 2003-2009 and a recent collection made for this study in 2015 . Our main objectives were to test for signals of reduced genetic diversity lasting from the intensive control efforts during the 1960s to the 1980s, and to determine if there have been statistically significant changes in allelic frequencies between temporally spaced samples made over a period of 12 years ( 96 generations). Results add to our understanding of the rate of evolutionary changes that influence G. pallidipes population dynamics. This information will help in making empirically derived vector control and monitoring recommendations for these regions in southwestern Kenya that have proven difficult to manage and that are at risk of reemergence of HAT from neighboring regions to the northwest.

\section{Methods}

\section{Study area and sampling strategy}

The samples used in this study were collected from Nguruman Escarpment and Ruma National Park in southwestern Kenya (Fig. 1). The Nguruman is a $1500 \mathrm{~km}^{2}$ area that lies north of the Kenya/Tanzania border that is managed for community use [31]. Recent control efforts consisted of deployment of 100 NG2B traps, which was reported to have led to a $99 \%$ reduction in G. pallidipes population density in 1987 [21], but the region has seen recent increase in population density because control programs stopped $[14,16,19]$. The other region of focus in this study, Ruma, is a $120 \mathrm{~km}^{2}$ protected area east of the Kenya/Uganda border that is managed for wildlife conservation [30]. Past control efforts included aerial and ground spraying, clearing of underbrush and use of insecticide treated targets from the 1960 s to the $1980 \mathrm{~s}[21,22]$, but the region has also seen recent increase in population density after control efforts reduced $[19,25]$.

This study combined samples collected from 2003 to 2009 from past studies [14, 19, 25], and newly collected samples from 2015. In all cases, G. pallidipes were
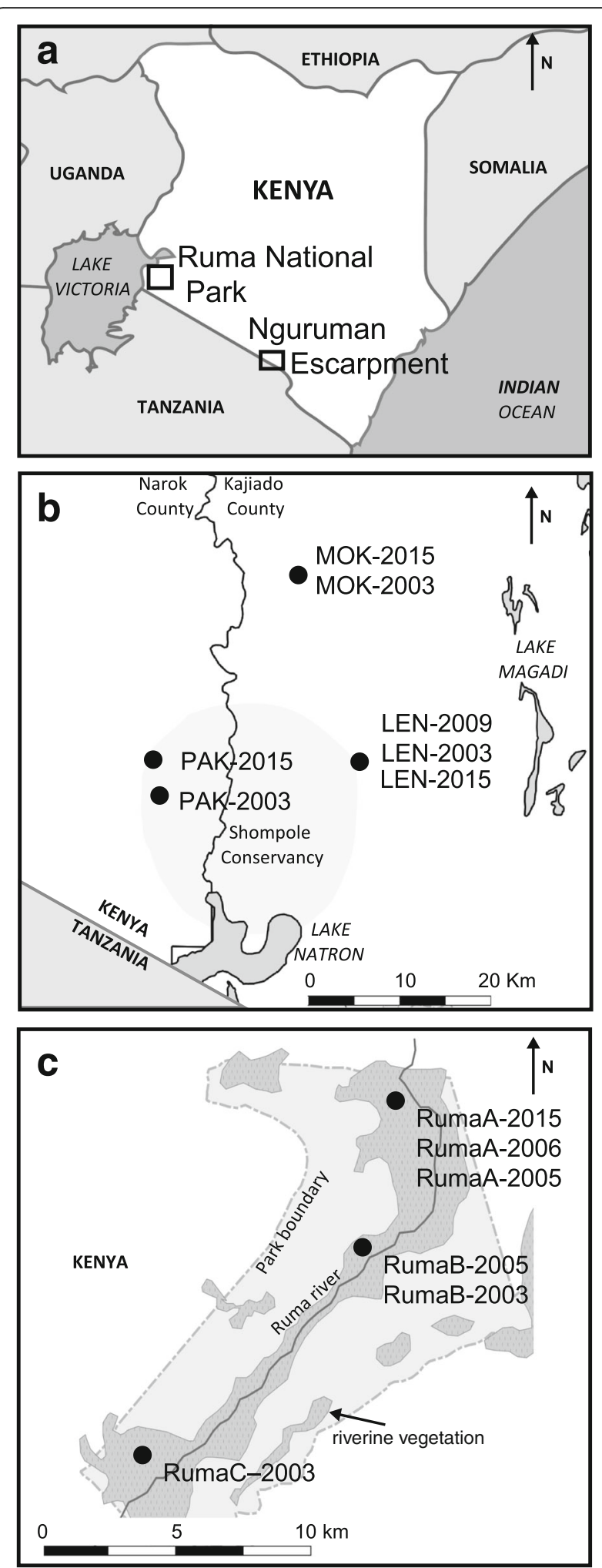

Fig. 1 Map of sampled regions southwestern Kenya (a), and sampled locations within Nguruman (b) and Ruma (c). Dots represent the sampling locations and are labeled by their abbreviation in Table 1 and the year of collection 
collected using bi-conical or $\mathrm{Ngu}$ traps that were set in clusters of 3-5 traps separated by less than $5 \mathrm{~km}$. Collected flies were preserved in $80 \%$ ethanol, transported for a maximum of 5 days at room temperature, and stored at $4{ }^{\circ} \mathrm{C}$. Later, a subsample was chosen for DNA extraction and microsatellite screening. For Nguruman, we screened an average of 38 individuals per site, collected from three locations spaced $<22 \mathrm{~km}$ apart (Fig. 1b). For Ruma, we screened an average of 20 individuals per site collected from the same three locations described by Ouma et al. [19] spaced $<14 \mathrm{~km}$ apart (Fig. 1c).

\section{DNA extraction and genotyping}

We extracted total DNA from two legs per fly using either the PrepGEM insect DNA (ZYGEM Corp Ltd., Hamilton, New Zealand) or the Qiagen DNAeasy blood and tissue (Qiagen, Hilden, Germany) extraction kits, following the manufacturer instructions. We used the Promega GoTaq kit (Madison, WI, USA) together with fluorescently-labeled primers for a $15 \mu \mathrm{l}$ PCR reaction as follows: $1 \mu \mathrm{l}$ of DNA template, $6 \mu \mathrm{l}$ of distilled $\mathrm{H}_{2} \mathrm{O}$, $2.6 \mu \mathrm{l}$ distilled Promega PCR Buffer, $0.1 \mu \mathrm{l}$ of $100 \times$ BSA (molecular grade), $0.5 \mu \mathrm{l}$ each of $10 \mathrm{mM}$ forward and reverse primers, $1.1 \mu \mathrm{l}$ of $25 \mathrm{mM} \mathrm{MgCl} 2,1.1 \mu \mathrm{l}$ of $10 \mathrm{mM}$ $\mathrm{dNTP}$ mix, and $0.1 \mu \mathrm{l}$ of $5 \mathrm{U} / \mu \mathrm{l}$ GoTaq DNA polymerase. Additional file 1: Tables S1 and S2 summarizes the microsatellite genotype strategy and protocol sources [32-35], and the multiplexing conditions. While GpB20b, GpC26b, GpA19a, GpB6b, GpC5b, GpC10b and GpCAG133 were developed for G. pallidipes [34] (Additional file 1: Table S1), the other six markers were developed for G. morsitans or G. fuscipes [32, 33, 35] (Additional file 1: Table S1) and have not all been validated for use in population genetic studies of G. pallidipes [32, 33, 35]. Four of these six markers, GmmK22, GmmA06, GmmC17 and GmmL11 $[33,36]$ have been tested for amplification in G. pallidipes $[22,34]$. Since the remaining two loci, D05 and Gmm8 $[32,35]$, had never been used in G. pallidipes prior to their inclusion in this study, we tested them in two individuals from four sampling sites using PCR amplification protocols used in other tsetse species [33, 35] to assess their reliability and variability. For all markers, PCR products were multiplexed in groups of two or three loci (Additional file 1: Table S2) together with $0.5 \mu \mathrm{l}$ of the Gel Co ROX internal size and Highly De-ionized Formamide (Gel Company, 665 Third street, San Francisco, CA, USA) to bring the total volume to $15 \mu \mathrm{l}$. The PCR products were then sized on an $\mathrm{ABI}$ 3730xL automated sequencer at the DNA Analysis Facility on Science Hill at Yale University (http://dna-analysis.yale.edu/). Alleles were scored using the program GeneMarker v 2.4.0 (Soft Genetics, Pennsylvania, USA). For all 13 markers, to ensure repeatability of genotype calls, we manually edited the automatically scored peaks twice independently following agreed upon binning rules (Additional file 1: Table S3), retaining only congruent genotype calls, which made up close to $100 \%$ of the peaks with amplification.

\section{Microsatellite marker validation, genetic diversity, and demographic estimates}

For all 13 markers, regardless of their history of use in population genetics studies in G. pallidipes, we conducted marker validation analysis to ensure that they did not contain null alleles or violate population genetics analysis assumptions. We tested for presence of null alleles using MICROCHECKER v2.2 [37], and for deviations from Hardy-Weinberg equilibrium (HWE) and for signals of linkage disequilibrium (LD) on a per-locus basis using Genepop v4.1 [38], and corrected for multiple tests with the Benjamini-Hochberg [39] method in both analyses.

To assess patterns of genetic diversity for all samples singly and grouped by time point, we estimated allele frequencies using GenAlex v6.502 [40], and allelic richness using FSTAT v2.9.3.2 [41]. Levels of observed $\left(\mathrm{H}_{\mathrm{O}}\right)$ and expected $\left(\mathrm{H}_{\mathrm{E}}\right)$ heterozygosity were estimated using Arlequin v3.5 [42]. Inbreeding coefficient $\left(\mathrm{F}_{\mathrm{IS}}\right)$ and deviations from HWE expectations were tested using FSTAT v2.9.3 [41] based on 10,000 randomizations. To understand the possible impact of the control efforts during the 1960 s to the 1980 s, and to identify recent demographic changes during the 12 year period studied, we estimated effective population size $\left(\mathrm{N}_{\mathrm{e}}\right)$ using the twosample temporal method [43] developed by Jorde \& Ryman [44] with the software NeESTIMATOR v2 [45]. We used the two-samples temporal method [45] because it is robust in the case of overlapping generations and can deal with low levels of polymorphisms [43, 44, 46], which are both concerns with this dataset.

We also evaluated the occurrence of recent bottlenecks for all samples singly and grouped by time point using the unilateral Wilcoxon test for heterozygosity excess implemented in the program BOTTLENECK v1.2.02 [47]. These tests were based on coalescence simulations at mutation-drift equilibrium under the two-phase model (TPM) and the infinite allele model (IAM), and used the mode-shift test [48] to detect deviations from the expected allele frequency distribution using the same program [47].

\section{Genetic differentiation among samples}

To assess genetic structure among samples, we used the Bayesian clustering method implemented in STRUCTURE v2.3.4 [49]. This method uses a Markov Chain Monte Carlo (MCMC) procedure to group individuals into genetic clusters $(\mathrm{K})$ that minimize deviation from HWE and reduce Linkage disequilibrium (LD) without a priori grouping. We allowed for admixture and inferred 
alpha in 10 independent runs for each $\mathrm{K}$ between 1 and 10 , using a 'burn-in' period of 50,000 followed by 250,000 MCMC steps. We visualized results for each $\mathrm{K}$ value using the online software CLUMPAK [50], and visualized the estimated likelihoods of the data from each run using the online software STRUCTURE HARVESTER [51]. We also visualized patterns of variation among samples with a principal component analysis (PCA) using centroids and ellipses that encompassing $95 \%$ of the variance within each sample. We also estimated a neighbor-joining tree [52] using Nei's genotype frequency-based distances [53] implemented in the "PopPR" v2.3.0 package $[55,56]$ in R with 1000 bootstrap replicates.

To assess levels of genetic differentiation among both geographically and temporally spaced samples, we estimated pairwise $\mathrm{F}_{\mathrm{ST}}$ in Arlequin v.3.5 [42] with Wright's statistics [56], following the variance method developed by Weir \& Cockerham [57] and using 10,000 permutations to obtain exact $P$-values. With the resulting $\mathrm{F}$ statistics converted to $\mathrm{F}_{\mathrm{ST}} /\left(1-\mathrm{F}_{\mathrm{ST}}\right)$, we tested for isolation by distance, both geographically $(\mathrm{km})$ and temporally (generations), using Rousset's procedure [58] implemented in the "isolation by distance" v3.23 web service [59]. Geographical distances were generated using the web-based "geographic matrix generator" v1.2.3 [60], and the significance of the regression was tested by a Mantel test with 10,000 randomizations [61]. Temporal distances were calculated in number of generations assuming 2.4 months per generation (5 generations per year [62-64]). Temporal and geographical distances between samples are reported in Additional file 1: Table S4. To assess differences in allelic and genotypic frequencies among geographically and temporally spaced samples, we calculated the per locus frequencies and carried out Fisher's exact tests between each sample [65] with 10,000 dememorizations, 1000 batches, and 10,000 iterations per batch, using Genepop [38]. Allele frequencies were visualized using 100\% stacked bar charts in Microsoft Excel 2010.

\section{Results}

Microsatellite marker validation, genetic diversity, and demographic estimates

MICROCHECKER indicated null alleles in GpB6B and Gmm8. Results from tests for per-locus deviations from HWE and LD are presented in Additional file 1: Table S5 and Additional file 1: Table S6. There were significant HWE deviations in two or more samples in GpB6B and Gmm8 (Additional file 1: Table S5) and since these loci also had indications of null alleles, they were removed from the final dataset. There was significant LD in both regions between loci D05 and GpC10b after the Benjamini Hochberg [39] correction (Additional file 1: Table S6), so D05 was removed from the final dataset. Only the 11 loci with no evidence of null alleles and no significant deviations from expectations of neutral evolution and Mendelian inheritance (GpC5b, CmmK22, GmmC17, GmmL11, GmmA06, GpB20b, GpC10b, GpA19a, GpC AG133, GpC26b) were used in subsequent analyses.

Summary diversity statistics for all samples are presented in Table 1, and allele frequencies in each sample are plotted in Additional file 1: Figure S1. For Nguruman, per sample estimates of allelic richness ranged between 3.2-3.8 and averaged 3.8 (Table 1), and were 3.6 in the pooled sample from 2015, 3.9 in the pooled sample from 2009, and 3.4 in the pooled sample from 2003. Expected $\left(\mathrm{H}_{\mathrm{E}}\right)$ and observed $\left(\mathrm{H}_{\mathrm{O}}\right)$ heterozygosity was similar among replicates between sampling points, with $\mathrm{H}_{\mathrm{O}}$ ranging between $0.406-0.504$ (Table 1 ). $\mathrm{F}_{\mathrm{IS}}$ estimates ranged bewteen $-0.085-0.210$ (Table 1) and were 0.033 in the pooled sample from 2015, 0.210 in the pooled sample from 2009, and -0.039 in the pooled sample from 2003, which indicates slight deviations from HWE expectations in 2003 and 2009. For Ruma, allelic richness ranged between 2.7-3.0 (Table 1), and were 3.7 in the pooled sample from 2015, 4.5 in the pooled sample from 2006, 4.0 in the pooled sample from 2005 , and 3.9 in the pooled sample from 2003. $\mathrm{H}_{\mathrm{E}}$ and $\mathrm{H}_{\mathrm{O}}$ estimates were similar among replicates between the sampling points, with $\mathrm{H}_{\mathrm{O}}$ ranging between 0.338-0.433 (Table 1). $\mathrm{F}_{\mathrm{IS}}$ estimates ranged between $0.025-0.228$ (Table 1), and were 0.117 in the pooled sample from $2015,-0.02$ in the pooled sample from 2006, 0.127 in the pooled sample from 2005, and 0.013 in the pooled sample from 2003, again indicating slight deviations from HWE expectations.

Tables 2 and 3 report the estimates of $\mathrm{N}_{\mathrm{e}}$ and results from tests for bottlenecks, respectively, from pooled samples from each region and year. The single-sample estimates can be found in Additional file 1: Table S7. For the Nguruman, $\mathrm{N}_{\mathrm{e}}$ estimates ranged between 144.0-731.7, with CIs ranging from an absolute low of 92.8 to an absolute high of 1067.2 (Table 2). BOTTLENECK results showed little indication of bottlenecks in any of the pooled samples (Table 3). Lenient tests under the IAM model showed a possible bottleneck in 2015 and 2003, but this was not supported by the analysis under the TPM model or by the mode-shift tests. For Ruma, $\mathrm{N}_{\mathrm{e}}$ estimates [44] ranged between 170.0-1684.89, with CIs ranging from an absolute low of 101.8, to an absolute high of 2572 (Table 2). BOTTLENECK results showed no indication of bottlenecks under the IAM or TPM models, or with the modeshift indicator test (Table 3). These results suggest no significant recent $\mathrm{N}_{\mathrm{e}}$ reductions in Ruma.

\section{Genetic differentiation among samples}

Results indicate there was no differentiation among geographically spaced samples in either Nguruman or 
Table 1 Summary statistics based on 13 microsatellite loci for G. pallidipes samples collected in Nguruman (LEN, MOK and PAK) by sample and pooled per year, and from Ruma (A, B and C) by sample and pooled per year

\begin{tabular}{|c|c|c|c|c|c|c|c|c|}
\hline Locality & Latitude & Longitude & Month/Year & $n$ & $A R$ & $\mathrm{H}_{\mathrm{O}}$ & $\mathrm{H}_{\mathrm{E}}$ & $F_{I S}$ \\
\hline \multicolumn{9}{|l|}{ Nguruman } \\
\hline LEN-2015 & 1.9769 & 36.1167 & February 2015 & 48 & 3.2 & 0.527 & 0.535 & 0.017 \\
\hline MOK-2015 & -1.8316 & 36.0893 & February 2015 & 53 & 3.7 & 0.499 & 0.525 & 0.049 \\
\hline PAK-2015 & -1.9804 & 35.9825 & February 2015 & 19 & 3.4 & 0.504 & 0.517 & 0.026 \\
\hline LEN-2009 & -1.9582 & 36.1196 & November 2009 & 29 & 3.8 & 0.406 & 0.511 & $0.210^{*}$ \\
\hline LEN-2003 & -1.9576 & 36.1199 & December 2003 & 30 & 3.2 & 0.534 & 0.493 & -0.085 \\
\hline MOK-2003 & -1.8246 & 36.0966 & December 2003 & 59 & 3.4 & 0.535 & 0.523 & -0.024 \\
\hline PAK-2003 & -2.0000 & 36.0000 & December 2003 & 29 & 3.2 & 0.524 & 0.508 & -0.032 \\
\hline 2015 (all sites) & na & na & February 2015 & 120 & 3.6 & 0.511 & 0.528 & 0.033 \\
\hline 2009 (all sites) & na & na & November 2009 & 29 & 3.9 & 0.406 & 0.511 & $0.210^{*}$ \\
\hline 2003 (all sites) & na & na & December 2003 & 118 & 3.4 & 0.533 & 0.513 & $-0.039^{*}$ \\
\hline \multicolumn{9}{|l|}{ Ruma } \\
\hline RumaA-2015 & -0.6082 & 34.3065 & November 2015 & 30 & 2.8 & 0.355 & 0.401 & $0.117^{*}$ \\
\hline RumaA-2006 & -0.6082 & 34.3065 & October 2006 & 30 & 3.2 & 0.453 & 0.446 & -0.015 \\
\hline RumaA-2005 & -0.6082 & 34.3065 & October 2005 & 10 & 2.7 & 0.422 & 0.425 & 0.007 \\
\hline RumaB-2005 & -0.6531 & 34.2677 & October 2005 & 10 & 2.8 & 0.398 & 0.474 & $0.168^{*}$ \\
\hline RumaC-2005 & -0.7005 & 34.2200 & October 2005 & 10 & 3.1 & 0.438 & 0.537 & $0.193^{*}$ \\
\hline RumaB-2003 & -0.6531 & 34.2677 & April 2003 & 30 & 3.1 & 0.446 & 0.452 & 0.013 \\
\hline 2015 & na & na & November 2015 & 30 & 3.7 & 0.355 & 0.401 & $0.117^{*}$ \\
\hline 2006 & na & na & October 2006 & 30 & 4.5 & 0.453 & 0.446 & -0.015 \\
\hline 2005 & na & na & October 2005 & 30 & 4.0 & 0.362 & 0.414 & $0.127^{*}$ \\
\hline 2003 & na & na & April 2003 & 30 & 3.9 & 0.446 & 0.452 & 0.013 \\
\hline
\end{tabular}

*Significant $\mathrm{F}_{\mathrm{IS}}$ values indicating deviations from Hardy-Weinberg equilibrium $(P$-value $<0.05)$

Abbreviations: $n$ number of samples analyzed, na not applicable, $A R$ mean allelic richness among all loci, $H_{O}$ observed heterozygosity, $H_{E}$ expected heterozygosity, $F_{I S}$ inbreeding coefficient

Table 2 Results for samples pooled by year for estimates of effective population size $\left(\mathrm{N}_{\mathrm{e}}\right)$ based on the Jorde/Ryman temporal method showing the interval between samples in number of generations (Interval), the $\mathrm{N}_{\mathrm{e}}$ estimate, the 95\% confidence interval (Cl)

\begin{tabular}{lllll}
\hline Region & Collections used & Interval & $\mathrm{N}_{\mathrm{e}}$ & $\mathrm{Cl}$ \\
\hline Nguruman (all sites) & $2003-2015$ & 55.8 & 300.6 & $197.4-425.3$ \\
Nguruman (all sites) & $2009-2015$ & 26.3 & 144.0 & $92.8-206.3$ \\
Nguruman (all sites) & $2003-2009$ & 29.6 & 731.7 & $459-1067.2$ \\
Ruma (all sites) & $2003-2015$ & 62.9 & 697.5 & $403.3-1071.3$ \\
Ruma (all sites) & $2005-2015$ & 50.4 & 1684.8 & $983.6-2572.7$ \\
Ruma (all sites) & $2006-2015$ & 45.4 & 401.9 & $242.2-601.5$ \\
Ruma (all sites) & $2003-2005$ & 12.5 & 426.3 & $248.9-650.9$ \\
Ruma (all sites) & $2003-2006$ & 17.5 & 1465.3 & $876.8-\infty$ \\
Ruma (all sites) & $2005-2006$ & 5.0 & 170.0 & $101.8-255.7$ \\
\hline
\end{tabular}

Table 3 Bottleneck test results bottleneck test results showing the $P$-values $(P)$ under the two-phase model (TPM), the infinite allele model (IAM) and using the mode-shift test (a result of normal $L$-shaped $(L)$ indicates no evidence of a bottleneck). All estimates of $\mathrm{N}_{\mathrm{e}}$ were made using the software NeESTIMATOR v2 [50], and all tests for population bottlenecks were made using the software BOTTLENECK v. 1.2.02 [55]

\begin{tabular}{lllll}
\hline Region & Collections used & TPM $P$ & IAM $P$ & Mode-Shift \\
\hline Nguruman (all sites) & 2015 & 0.500 & $0.009^{*}$ & $\mathrm{~L}$ \\
Nguruman (all sites) & 2009 & 0.539 & 0.188 & $\mathrm{~L}$ \\
Nguruman (all sites) & 2003 & 0.539 & $0.042^{*}$ & $\mathrm{~L}$ \\
Ruma (all sites) & 2015 & 0.787 & 0.545 & $\mathrm{~L}$ \\
Ruma (all sites) & 2006 & 0.947 & 0.722 & $\mathrm{~L}$ \\
Ruma (all sites) & 2005 & 0.784 & 0.500 & $\mathrm{~L}$ \\
Ruma (all sites) & 2003 & 0.820 & 0.326 & $\mathrm{~L}$ \\
\hline${ }^{*}<<0.05$ & & & &
\end{tabular}



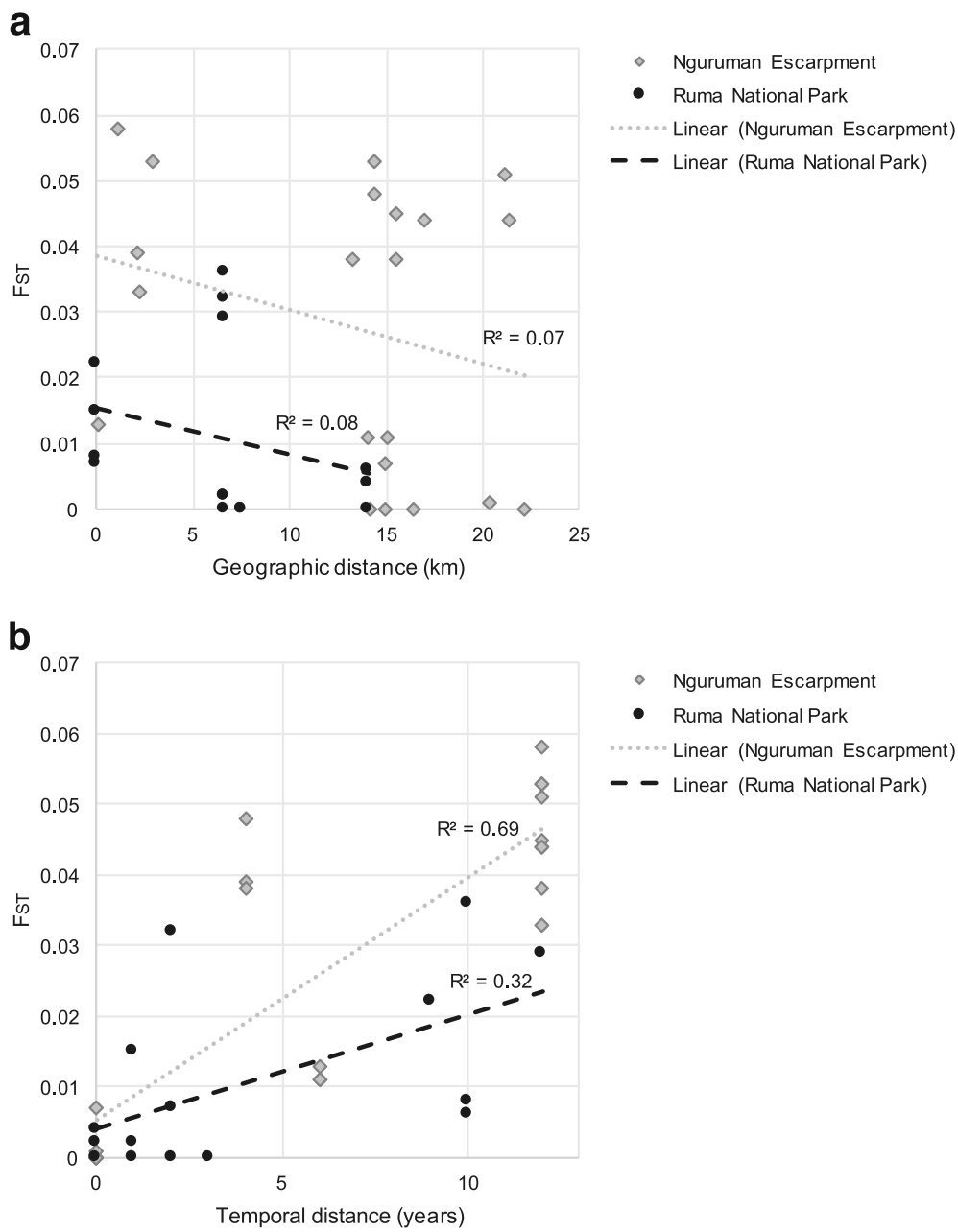

Fig. 2 Results of tests for isolation by distance showing the linear regression of genetic differentiation $\mathrm{F}_{S T} /\left(1-\mathrm{F}_{\mathrm{ST}}\right)$ by geographical distance ( $\mathrm{km}$ ) (a), and temporal distance (generations) (b), with the best line of linear fit and $\mathrm{R}^{2}$ values displayed by region. Tests for isolation by distance were completed using Rousset's procedure [63] implemented in the "isolation by distance" v3.23 web service [64]. Geographical distances were generated using the web-based "geographic matrix generator" $\vee 1.2 .3[65]$, and the significance of the regression $(P)$ and strength of the correlation $\left(R^{2}\right)$ were tested by a Mantel test with 10,000 randomizations [66]

Ruma. This is suggested by the results of the Bayesian clustering analyses implemented using STRUCTURE (Additional file 1: Figure S2), and the PCA plots (Additional file 1: Figure S3), and by the NJ tree, which showed that samples from the same location do not form separate monophyletic groups Additional file 1: Figure S4. Lack of geographical structure is also supported by low $\mathrm{F}_{\mathrm{ST}}$ estimates among geographically separated samples from the same years (Table 3), and no correlation between genetic and geographical distances (Nguruman: $R^{2}=0.01, P$-value $=0.748$; Ruma: $R^{2}=0.08$, $P$-value $=0.820 ;$ Fig. $2 \mathrm{a})$. Furthermore, there were no significant allelic/genotypic differences among geographically spaced samples from the same years (Table 6).

In contrast, there was strong evidence of genetic differentiation among temporally spaced samples in Nguruman but much weaker one among Ruma samples.
The neighbor-joining tree grouped all 2015 samples together in $49.3 \%$ of bootstrap replicates and all 2003 samples together in $63.7 \%$ of bootstrap replicates (Additional file 1: Figure S4a). $\mathrm{F}_{\mathrm{ST}}$ estimates ranged bewteen -0.003-0.059 (Table 3), with significant temporal differentiation between the 2015 samples and all other time points (Table 3). The Mantel test indicated significant correlation of genetic and temporal distance with a slope of 9.88e-4 $\left(R^{2}=0.70, P\right.$-value $=0.013$, Fig. $\left.2 \mathrm{~b}\right)$. Furthermore, Fisher's exact tests comparing allelic and genotypic frequencies indicated that 13 out of 15 temporally spaced samples were significantly different from one another (Table 6), and none out of 6 geographically spaced samples were significantly different from one another (Table 6).

For Ruma, the neighbor joining tree did not cluster samples by year of collection (Additional file 1: Figure 
Table 4 Pairwise FST values calculated in ARLEQUIN v.3.5 [47] with Wright's statistics [56], following the variance method developed by Weir \& Cockerham [57] and using 10,000 permutations to obtain exact $P$-values [61] from Nguruman

\begin{tabular}{llllllll}
\hline & LEN-2015 & MOK-2015 & PAK-2015 & LEN-2009 & LEN-2003 & MOK-2003 & PAK-2003 \\
\hline LEN-2015 & - & & & & & \\
MOK-2015 & $0.001^{\mathrm{a}}$ & - & & & & \\
PAK-2015 & $-0.003^{\mathrm{a}}$ & $-0.001^{\mathrm{a}}$ & - & & & \\
LEN-2009 & $0.042^{*}$ & $0.045^{*}$ & $0.053^{*}$ & - & & \\
LEN-2003 & $0.042^{*}$ & $0.058^{*}$ & $0.059^{*}$ & 0.012 & - & \\
MOK-2003 & $0.039^{*}$ & $0.050^{*}$ & $0.048^{*}$ & 0.008 & $-0.001^{\mathrm{a}}$ & - & $-0.001^{\mathrm{a}}$ \\
PAK-2003 & $0.041^{*}$ & $0.057^{*}$ & $0.048^{*}$ & 0.007 & $0.003^{\mathrm{a}}$ & - \\
\hline
\end{tabular}

*Significant $P$-values after Benjamini-Hochberg correction [44]

${ }^{\mathrm{a} C o m p a r i s o n}$ of two samples from the same year

S4). $\mathrm{F}_{\mathrm{ST}}$ estimates were low (Table 4), ranging between $-0.017-0.036$ (Table 4), with significant differentiation between 2003 and 2015 samples, some 2005 and 2006 samples, and 2003 and 2005 samples (Table 4). There was weak indication of differentiation among temporally spaced samples that did not create a significant pattern of isolation by temporal distance, as the Mantel tests indicated no correlation of genetic and temporal distances with a slope of 5.94e- $4\left(R^{2}=0.05, P\right.$-value $=0.277$, Fig. 2b). Low levels of differentiation among temporal samples is also supported by the Fisher's exact tests comparing allelic and genotypic frequencies.

\section{Discussion}

\section{Genetic diversity and demographic estimates}

Allelic richness estimates indicate that Nguruman and Ruma exhibited levels of genetic diversity in samples pooled by year were similar to each other (ranging between 3.4-4.5; Table 1) and to those reported throughout the G. pallidipes distribution (ranging between 3.2-5.6 [25]). Allelic richness across both regions averaged 3.8 (Table 1), suggesting that genetic diversity was not substantially reduced by the control efforts during the 1960s to the1980s. This could be due to a variety of reasons that could have acted separately or together. It could be that the control measures were not maintained for long enough to impact the genetic diversity. Tsetse flies have relatively long life span compared to other insect vectors [66], and this could have allowed large enough numbers to survive in local relict populations, so that genetic diversity was maintained "on site". This possibility is supported by positive $F_{\text {IS }}$ estimates in 2009 in Nguruman, and in 2015 and 2005 in Ruma (Table 1). Positive $F_{I S}$ indicates heterozygotes excess and is consistent with rapid demographic fluctuations and local geographical retractions and expansions [67]. Another possible explanation for the relatively high allelic richness could be recolonizations from neighboring regions after the control measures were discontinued. Both Nguruman and Ruma are geographically close to high tsetse densities in neighboring countries, making recolonizations from outside regions a real possibility. Indeed, a mix of individuals from different genetic backgrounds (i.e. the Wahlund effect) could also cause the finding of positive $\mathrm{F}_{\mathrm{IS}}$ estimates (Table 1). Taken together, previous spatial analyses $[16,25]$ and the data presented here cannot distinguish between these possible scenarios.

$\mathrm{N}_{\mathrm{e}}$ estimates were similar to those from other studies either on the same $[20,25]$ or a closely related species, G. fuscipes fuscipes [68-73]. Estimates comparable to other populations without a history of strong vector control suggest that the population reduction during the control efforts of the 1960s to the1980s either did not result in significant loss of genetic diversity or changes in allele frequencies, or that the populations rebounded quickly to pre-control effective population sizes. Future work with densely distributed markers such as Single Nucleotide Polymorphisms (SNPs) combined with the use of model based approaches to estimate demographic parameters, such as Approximate Bayesian Computation [74], could provide more precise $\mathrm{N}_{\mathrm{e}}$ estimates.

Results from tests for bottlenecks do not provide strong evidence of significant genetic bottlenecks in either region. Although the methods used can only detect extreme reductions in population sizes that occurred more recently than $2-4 \mathrm{~N}_{\mathrm{e}}$ generations ago $[75,76]$, they are sensitive enough to have detected genetic bottlenecks in G. pallidipes in previous studies [20, 75]. In tsetse flies, such as G. pallidipes, severe bottlenecks can probably be detected as far back as $\sim 1400$ generations, or 175 years, assuming $\mathrm{N}_{\mathrm{e}}$ is close to the mean of $\sim 700$, as reported in other studies [68-73], and that the average number of generations is close to 5 per year [62-64]. Indead, none of the tests in samples grouped by year were significant in Ruma (Table 2), none of the tests using the most appropriate TPM model [47] were significant, and only two single time-point samples showed a signal of a bottleneck using the lenient IAM model (Table 3). Thus, indications of local bottlenecks are non- 
Table 5 Pairwise FST values calculated in ARLEQUIN v.3.5 [47] with Wright's statistics [56], following the variance method developed by Weir \& Cockerham [57] and using 10,000 permutations to obtain exact $P$-values [61] from Ruma

\begin{tabular}{lllllll}
\hline & RumaA-2015 & RumaA-2006 & RumaA-2005 & RumaB-2005 & RumaC-2005 & RumaB-2003 \\
\hline RumaA-2015 & - & & & & \\
RumaA-2006 & $0.029^{*}$ & - & & & \\
RumaA-2005 & 0.006 & $0.028^{*}$ & - & - & - \\
RumaB-2005 & $0.034^{*}$ & 0.006 & $0.018^{\mathrm{a}}$ & $-0.017^{\mathrm{a}}$ & -0.009 \\
RumaC-2005 & 0.001 & -0.010 & $0.017^{\mathrm{a}}$ & 0.004 & - \\
RumaB-2003 & $0.022^{*}$ & -0.003 & $0.036^{*}$ & & - \\
\hline
\end{tabular}

*Significant $P$-values after Benjamini-Hochberg correction [44]

${ }^{a}$ Comparison of two samples from the same year

conclusive, and may simply reflect strong genetic drift in Nguruman.

\section{Genetic differentiation among samples}

To evaluate the long-term population dynamics of $G$. pallidipes, we assessed levels and patterns of geographical and temporal genetic differentiation among samples from the two regions. We found no significant $\mathrm{F}_{\mathrm{ST}}$ estimates or differnces in allelic or genotypic frequencies among geographically spaced samples collected during the same time interval in each region (Tables 4, 5), and no evidence of isolation by geographical distance (Fig. 2a). Although not unexpected, given the small geographical scale (Fig. 1) and levels of differentiation reported in other G. pallidipes studies [19], this result is different from the findings in the closely related species, G. F. fuscipes, where significant $\mathrm{F}_{\mathrm{ST}}$ estimates were found at a spatial distance of just $5 \mathrm{~km}[54,55]$. The lack of geographical structure found in this study suggests either that G. pallidipes moves greater distances than G. F. fuscipes, or that the external environment in Nguruman and Ruma have facilitated gene flow in these regions.

We identified significant temporal genetic differentiation in Nguruman, but not in Ruma. In Nguruman, we found significant genetic differentiation between samples collected before and after 2009 (Tables 4, 5; Additional file 1: Figure S4a) and a significant and tight correlation between genetic differentiation and temporal distance (Fig. 2b). These results are at odds with previous microsatellite based analyses in G. pallidipes by Ouma \& Krafsur [16] and Ouma et al. [19], and in G. fuscipes fuscipes $[56,57]$, which did not detect genetic differentiation between temporal samples. The difference between these studies is probably due to a number of factors, one of which is the longer period covered in our study (12 years; $\sim 96$ generations) as compared to Ouma \& Krafsur's [18] and Ouma et al.'s [21] studies (3-12 months; $~ 2-8$ generations), and Opiro et al.'s [56, 57] study (7 years,; 56 generations). Another factor may be the timing of collections relative to population disruptions. For example,
Ouma \& Krafsur [18] focused on samples collected from Nguruman in 2001-2003 and did not find any differentiation, while our samples from the same locality from 2003 to 2015 showed significant differentiation. These findings indicate that temporal genetic differentiation occurs in G. pallidipes and can be detected at a scale of $>80$ generations under some conditions. These findings also suggest that previous studies could not detect temporal differnetioan in Nguruman [16] because samples were collected too close in time to each other, and thus, future population genetic schemes should include a broad time frame to better detect temporal genetic differentiaion in tsetse.

\section{Implications for vector control}

Genetic diversity, $\mathrm{N}_{\mathrm{e}}$, and tests for bottlenecks indicate a similar response to intensive control efforts between the 1960s and the1980s between Nguruman and Ruma. There was no evidence of significantly reduce genetic diversity, and estimates were similar to those reported in other regions without a history of intensive control measures $[20,25]$. Furthermore, results from the bottleneck tests did not indicate genetic bottlencks. These findings suggest that for G. pallidipes, even massive control efforts have failed to have a long-term influence on genetic diversity and allele frequency. This could be because control measures were not maintained for long enough to impact the genetic diversity, or because there were recolonizations from neighboring regions after the control measures were discontinued.

However, we did find differences in the results of the bottleneck tests and levels of temporal genetic differentiation between these two regions. Nguruman displayed higher levels of differentiation (Fig. 2; Additional file 1: Fig. S4) and larger changes in allele/genotype frequencies between temporally spaced samples (Table 6). Differences could suggest that populations in these regions responded to the control campaign and its halting in different ways, or that populations in these regions were exposed to different conditions after the control 
Table 6 Fisher's exact test for genotypic and allelic differentiation of 13 microsatellites from Nguruman and Ruma, organized by locality and year of collection, showing the comparison, the sample pair used in the test

\begin{tabular}{|c|c|c|c|c|c|c|c|}
\hline \multirow[t]{2}{*}{ Comparison } & \multirow[t]{2}{*}{ Sample pair } & \multicolumn{3}{|c|}{ Genotypic } & \multicolumn{3}{|c|}{ Allelic } \\
\hline & & $\overline{x^{2}}$ & $d f$ & $P$-value & $x^{2}$ & $d f$ & $P$-value \\
\hline \multicolumn{8}{|l|}{ Nguruman } \\
\hline \multirow[t]{3}{*}{ Within LEN } & LEN-2015 \& LEN-2009 & $\infty$ & 20 & $<0.001^{*}$ & $\infty$ & 20 & $<0.001^{*}$ \\
\hline & LEN-2015 \& LEN-2003 & $\infty$ & 20 & $<0.001^{*}$ & $\infty$ & 20 & $<0.001^{*}$ \\
\hline & LEN-2009 \& LEN-2003 & 27.2 & 20 & 0.131 & 23.0 & 20 & 0.287 \\
\hline \multirow[t]{6}{*}{ LEN \& MOK } & LEN-2015 \& MOK-2015 & 30.0 & 20 & 0.070 & 26.8 & 20 & 0.130 \\
\hline & LEN-2015 \& MOK-2003 & $\infty$ & 20 & $<0.001^{*}$ & $\infty$ & 20 & $<0.001^{*}$ \\
\hline & LEN-2009 \& MOK-2015 & $\infty$ & 20 & $<0.001^{*}$ & $\infty$ & 20 & $<0.001^{*}$ \\
\hline & LEN-2009 \& MOK-2003 & 34.6 & 20 & 0.022 & 32.5 & 20 & 0.038 \\
\hline & LEN-2003 \& MOK-2015 & $\infty$ & 20 & $<0.001^{*}$ & $\infty$ & 20 & $<0.001^{*}$ \\
\hline & LEN-2003 \& MOK-2003 & 18.3 & 20 & 0.569 & 18.7 & 20 & 0.540 \\
\hline \multirow[t]{6}{*}{ LEN \& PAK } & LEN-2015 \& PAK-2015 & 13.7 & 20 & 0.847 & 13.1 & 20 & 0.872 \\
\hline & LEN-2015 \& PAK-2003 & $\infty$ & 20 & $<0.001^{*}$ & $\infty$ & 20 & $<0.001^{*}$ \\
\hline & LEN-2009 \& PAK-2003 & $\infty$ & 20 & $<0.001^{*}$ & $\infty$ & 20 & $<0.001^{*}$ \\
\hline & LEN-2009 \& PAK-2015 & 23.3 & 20 & 0.272 & 22.1 & 20 & 0.334 \\
\hline & LEN-2003 \& PAK-2015 & $\infty$ & 20 & $<0.001^{*}$ & $\infty$ & 20 & $<0.001^{*}$ \\
\hline & LEN-2003 \& PAK-2003 & 18.5 & 20 & 0.556 & 20.1 & 20 & 0.448 \\
\hline Within MOK & MOK-2015 \& MOK-2003 & $\infty$ & 20 & $<0.001^{*}$ & $\infty$ & 20 & $<0.001^{*}$ \\
\hline \multirow[t]{4}{*}{ MOK \& PAK } & MOK-2015 \& PAK-2015 & 14.2 & 20 & 0.818 & 16.3 & 20 & 0.701 \\
\hline & MOK-2015 \& PAK-2003 & $\infty$ & 20 & $<0.001^{*}$ & $\infty$ & 20 & $<0.001^{*}$ \\
\hline & MOK-2003 \& PAK-2015 & $\infty$ & 20 & $<0.001^{*}$ & $\infty$ & 20 & $<0.001^{*}$ \\
\hline & MOK-2003 \& PAK-2003 & 21.2 & 20 & 0.383 & 22.6 & 20 & 0.307 \\
\hline Within PAK & PAK-2015 \& PAK-2003 & $\infty$ & 20 & $<0.001^{*}$ & $\infty$ & 20 & $<0.001^{*}$ \\
\hline \multicolumn{8}{|l|}{ Ruma } \\
\hline \multirow[t]{3}{*}{ within Block A } & RumaA-2015 \& RumaA-2006 & 43.6 & 20 & $0.002^{*}$ & 37.2 & 20 & $0.011^{*}$ \\
\hline & RumaA-2015 \& RumaA-2005 & 22.2 & 20 & 0.329 & 26.1 & 20 & 0.164 \\
\hline & RumaA-2006 \& RumaA-2005 & 20.6 & 20 & 0.418 & 18.6 & 20 & 0.547 \\
\hline \multirow[t]{6}{*}{ Block A \& B } & RumaA-2015 \& RumaB-2005 & $\infty$ & 18 & $<0.001^{*}$ & $\infty$ & 18 & $<0.001^{*}$ \\
\hline & RumaA-2015 \& RumaB-2003 & 37.8 & 18 & $0.004^{*}$ & 34.0 & 18 & $0.012^{*}$ \\
\hline & RumaA-2006 \& RumaB-2005 & 12.0 & 20 & 0.918 & 9.8 & 20 & 0.971 \\
\hline & RumaA-2006 \& RumaB-2003 & 14.7 & 20 & 0.796 & 14.5 & 20 & 0.803 \\
\hline & RumaA-2005 \& RumaB-2005ª & 22.2 & 20 & 0.331 & 18.9 & 20 & 0.529 \\
\hline & RumaA-2005 \& RumaB-2003 & 25.3 & 20 & 0.188 & 27.5 & 20 & 0.121 \\
\hline \multirow[t]{3}{*}{ Block A \& C } & RumaA-2015 \& RumaC-2005 & 25.5 & 18 & 0.111 & 22.2 & 18 & 0.221 \\
\hline & RumaA-2006 \& RumaC-2005 & 12.0 & 20 & 0.915 & 13.3 & 20 & 0.862 \\
\hline & RumaA-2005 \& RumaC-2005 & 19.0 & 20 & 0.519 & 15.2 & 20 & 0.767 \\
\hline within Block B & RumaB-2005 \& RumaB-2003 & 14.1 & 18 & 0.722 & 15.2 & 18 & 0.649 \\
\hline \multirow[t]{2}{*}{ Block B \& C } & RumaB-2005 \& RumaC-2005 & 8.7 & 18 & 0.966 & 7.7 & 18 & 0.983 \\
\hline & RumaB-2003 \& RumaC-2005 & 12.0 & 18 & 0.847 & 12.8 & 18 & 0.802 \\
\hline
\end{tabular}

${ }^{a}$ Comparison of two samples from the same year

${ }^{*} P<0.05$ 
measures were halted. The most obvious difference between the regions is that Ruma is protected from human use, while Nguruman is not. This different status has contributed to land-use alterations in Nguruman, including construction of irrigation systems and deforestation, as land was converted for cattle grazing and agriculture [26-29]. In addition,Nguruman has also been heavily impacted by Maasai animal grazing and trade, with cattle being brought in from as far as Lake Natron in Northern Tanzania [77, 78]. It is possible that these human activities may have disrupted the G. pallidipes habitats in Nguruman in ways that did not occur in Ruma, where land is fully protected from human use. We suggest that alteration of tsetse habitats due to human use [26-29] may be one of the main drivers for the temporal genetic changes detected in Nguruman. Another possibility that cannot be assessed with this dataset was continuous low levels of gene flow from outside areas druing the period studied that drove changes in allele and genotype frequencies observed. An important next step in understanding the drivers of temporal differentiation in Ngurman will be to assess genetic variation within a larger geographical context and with more advanced statistical approaches. For example, methods such as isolation by resistance $[79,80]$ and by environment [81] can help assess the impact of land use transformation on genetic data, by providing a way to disentangle the effect of geographical and ecological differentiation on genetic differentiation [82].

\section{Conclusions}

Our findings suggest that for G. pallidipes, even massive control efforts have not had lasting influence on genetic diversity, and that temporal genetic differentiation occurs in G. pallidipes under some circumstances, such as human land use. These findings are important because they enhance our understanding of the process underlying the local persistence of G. pallidipes populations in southwestern Kenya over the past four decades, stress the importance of long-term monitoring and sustained control efforts in control of G. pallidipes in southwestern Kenya, and suggest that different habitat management can play a significant role in shaping levels of genetic differentiation in this vector. In addition, they also point out important knowledge gaps which can be addressed by future research, such as the need of densely spaced genomic markers and of a sampling design that includes both target and neighboring populations. This would facilitate estimates of key demographic parameters and reconstructions of recent and past gene flow levels and patterns among different sampling sites. Improved estimates will increase our ability to identify the ecological, evolutionary, and/or human-mediated process underlying the genetic changes between temporally spaced G. pallidipes samples.

\section{Additonal file}

Additional file 1: Table S1. Microsatellite locus name, dye used, primer sequences, size range of the amplified product, repeat motif length and the original publication sourced for primer design. Table S2. Microsatellite multiplex name, and the locus marked with each dye. Table S3. GeneMarker panel binning rules, including multiplex design, upper and lower size (bp) boundaries for each marker, allele names, and binning center, minimum, and maximum. Table S4. Pairwise geographical distance $(\mathrm{km})$ and temporal distance (years) between samples from (a) Nguruman, and (b) Ruma. Table S5. Per locus $F_{15}$ values obtained after testing for deviation from Hardy-Weinberg equilibrium (HWE) using FSTAT v2.9.3 [46] based on 10,000 randomizations for (a) Nguruman and (b) Ruma. Table S6. Pairwise locus $P$-values after testing for linkage disequilibrium estimated in Genepop v4.1 [43]. Table S7. Per-sample (a) estimates of effective population size $\left(\mathrm{N}_{\mathrm{e}}\right)$ based on the Jorde/Ryman temporal method showing the interval used in generations (I), the $\mathrm{N}_{e}$ estimate, the $95 \%$ $\mathrm{Cl}$, and (b) bottleneck results showing the $P$-values $(P)$ under the two-phase model (TPM), the infinite allele model (IAM) and using the mode-shift test. Figure S1. Allele frequency of each of the 10 microsatellites from (a) Nguruman and (b) Ruma. Figure S2. STRUCTURE [54] estimated Ln likelihood of the data for K values 1-10 for (a) Nguruman and (b) Ruma, and plots of structure assignment for K values 2-10 for (c) Nguruman and (d) Ruma. Figure S3. Principal Components Analysis (PCA) of microsatellite data from (a) Nguruman and (b) Ruma. Figure S4. Neighbor-joining tree [57] using Nei's genotype frequency based distances (a) Nguruman and (b) Ruma. (DOCX 1088 kb)

\section{Abbreviations}

AAT: Animal African Trypanosomiasis; BSA: Bovine serum albumin; DAPC: Discriminant Analysis of Principal Components;

dNTP: Deoxynucleotide triphosphate; $F_{C T}$ : Variance among groups relative to the total variance; $F_{I S}$ : Inbreeding coefficient; $F_{I T}$ : Variance within individuals; $F_{S C}$ : Variance among subpopulations within groups; $F_{S T}$ : Variance among subpopulations relative to the total variance; HAT: Human African

Trypanosomiasis; $\mathrm{H}_{\mathrm{e}}$ : Expected heterozygosity; $\mathrm{H}_{\mathrm{o}}$ : Observed heterozygosity; HWE: Hardy-Weinberg equilibrium; IAEA: International Atomic Energy Agency; LEN: Lengong; MOK: Mukinyo; $\mathrm{N}_{\mathrm{e}}$ : Effective population size; PAK: Pakase; TPM: Two-phase model; WHO: World Health Organization

\section{Acknowledgements}

We would like to acknowledge Joanna Auma, Paul Thande, Patrick Obore, Lillian Mwende Mwaniki, Rose Wanjiru Ndung'u, and all of Biotechnology Research Institute, and the Kenya Wildlife Services for the immense amount of input and assistance in sample collection and DNA extraction. We also acknowledge Carol Mariani of the DNA Analysis Facility at Yale University for genotyping of the samples. We would also like to thank two anonymous reviewers. Their thoughtful comments have greatly improved the manuscript. The research was accomplished while WAO was a Research Fellow at Yale University.

\section{Funding}

NIH Grant no. U01 Al115648; NIH-Fogarty Global Infectious Diseases Training Grant (D43TW007391).

\section{Availability of data and materials}

Raw data are available in STRUCTURE format at https://datadryad.org with the doi: $10.5061 /$ dryad.8nd84.

\section{Authors' contributions}

Conceptualization: WAO, MM, POM, JO, GM, SA and AC. Sample collection: WAO, RB, POM, SO, JOO and GM. Sample processing: WAO, MM, KD and RB. Data generation: WAO, MM, KD and NPS. Data analysis: WAO, MM, NPS and KD. Manuscript development: WAO, NPS, MM and AC, Manuscript review: NPS, MM, KD, RB, POM, SO, JOO, CO, JO, GM, SA and AC. Supervision: NPS, $\mathrm{CO}, \mathrm{JO}, \mathrm{GM}, \mathrm{SA}$ and AC. All authors read and approved the final manuscript.

Ethics approval and consent to participate Not applicable

Consent for publication

Not applicable 


\section{Competing interests}

The authors declare that they have no competing interests.

\section{Publisher's Note}

Springer Nature remains neutral with regard to jurisdictional claims in published maps and institutional affiliations.

\section{Author details}

${ }^{1}$ Yale School of Public Health, Yale University, New Haven, CT, USA.

${ }^{2}$ Biotechnology Research Institute, Kenya Agricultural and Livestock Research Organization, Nairobi, Kikuyu, Kenya. ${ }^{3}$ Department of Biomedical Science and Technology, School of Public Health and Community Development, Maseno University, Kisumu, Maseno, Kenya. ${ }^{4}$ Department of Ecology \& Evolutionary Biology, Yale University, New Haven, CT, USA. ${ }^{5}$ Centre for Geographic Medicine Research Coast, Kenya Medical Research Institute, Kilifi, Kenya. ${ }^{6}$ Africa Technical Research Center, Vector Health International, Arusha, Tanzania. ${ }^{7}$ Centre for Biotechnology and Bioinformatics, University of Nairobi, Nairobi, Kenya.

Received: 29 December 2016 Accepted: 1 October 2017 Published online: 10 October 2017

\section{References}

1. WHO. Control and surveillance of human African Trypanosomiasis. WHO Technical Report Series; no. 2013:984. http://www.ncbi.nlm.nih.gov/ pubmed/24552089

2. FAO. Tsetse transmitted trypanosomiasis. FAO Corporate Document Repository. http://www.fao.org/wairdocs/ilri/x5539e/x5539e08.htmtheproblem.

3. CDC. Parasites - Africa Trypanosomiasis (also known as Sleeping Sickness). Centers for Disease Controls and Prevention 2012. https://www.cdc.gov/ parasites/sleepingsickness/treatment.html.

4. Aksoy S. Control of tsetse flies and trypanosomes using molecular genetics. Vet Parasitol. 2003;115:125-45.

5. Anene BM, Onah DN, Nawa Y. Drug resistance in pathogenic African trypanosomes: what hopes for the future? Vet Parasitol. 2001;96:83-100.

6. Wilkinson SR, Kelly JM. Trypanocidal drugs: mechanisms, resistance and new targets. Expert Rev Mol Med. 2009;11:e31.

7. Maudlin I. African trypanosomiasis. Ann Trop Med Parasitol. 2006;100:679-701.

8. Schofield CJ, Kabayo JP. Trypanosomiasis vector control in Africa and Latin America. Parasit Vectors. 2008;1:24.

9. Torr SJ, Hargrove JW, Vale GA. Towards a rational policy for dealing with tsetse. Trends Parasitol. 2005;21:537-41.

10. Vreysen MJ. Principles of area-wide integrated tsetse fly control using the sterile insect technique. Med Trop. 2001;61:397-411.

11. Rutto JJ, Karuga JW. Temporal and spatial epidemiology of sleeping sickness and use of geographical information system (GIS) in Kenya. J Vector Borne Dis. 2009:46:18-25.

12. Menon S, Rossi R, Nshimyumukiza L, Zinszer K. Revisiting zoonotic human African trypanosomiasis control in Uganda. J Public Heal Policy. 2017;37:51-67.

13. Hamill L, Picozzi K, Fyfe J, von Wissmann B, Wastling S, Wardrop N, et al. Evaluating the impact of targeting livestock for the prevention of human and animal trypanosomiasis, at village level, in districts newly affected with T. b. rhodesiense in Uganda. Infect Dis Poverty. 2017;6:16.

14. Ouma JO, Marquez JG, Krafsur ES. Macrogeographic population structure of the tsetse fly, Glossina pallidipes (Diptera: Glossinidae). Bull Entomol Res. 2005;95:437-47.

15. Williams B, Dransfield R, Brightwell R. Monitoring tsetse fly populations. I. The intrinsic variability of trap catches of Glossina pallidipes at Nguruman, Kenya. Med Vet Entomol. 1990;4:167-79.

16. Ouma JO, Krafsur ES. The influence of temporal and seasonal changes on genetic diversity and population structure of the tsetse fly, Glossina pallidipes in Kenya. East African Agric Forum J. 2010;77:59-68.

17. Solano P, Kaba D, Ravel S, Dyer NA, Sall B, Vreysen MJ, et al. Population genetics as a tool to select tsetse control strategies: suppression or eradication of Glossina palpalis gambiensis in the Niayes of Senegal. PLoS Negl Trop Dis. 2010;e692:4

18. Solano P, Ravel S, de Meeus T. How can tsetse population genetics contribute to African trypanosomiasis control? Trends Parasitol. 2010;26:255-63.
19. Ouma JO, Marquez JG, Krafsur ES. Microgeographical breeding structure of the tsetse fly, Glossina pallidipes in south-western Kenya. Med Vet Entomol. 2006;20:138-49

20. Ciosi M, Masiga DK, Turner CM. Laboratory colonisation and genetic bottlenecks in the tsetse fly Glossina pallidipes. PLoS Negl Trop Dis. 2014;8: e2697.

21. Dransfield RD, Brightwell R. C. K, Williams B. Control of tsetsefly (Diptera: Glossinidae) populations using traps at Nguruman, south-west Kenya. Bull Entomol Res. 1990:80:265-76.

22. Muriuki GW, Njoka TJ, Reid RS, Nyariki DM. Tsetse control and land-use change in Lambwe valley, south-western Kenya. Agric Ecosyst Environ. 2005; 106:99-107.

23. Wellde BT, Waema D, Chumo DA, Reardon MJ, Oloo F, Njogu AR, et al. Review of tsetse control measures taken in the Lambwe Valley in 19801984. Ann Trop Med Parasitol. 1989:83(Suppl 1):119-25.

24. Nesbitt SA, Gooding RH, Rolseth BM. Genetic variation in two field populations and a laboratory colony of Glossina pallidipes (Diptera: Glossinidae). J Med Entomol. 1990;27:586-91.

25. Ouma JO, Beadell JS, Hyseni C, Okedi LM, Krafsur ES, Aksoy S, et al. Genetic diversity and population structure of Glossina pallidipes in Uganda and western Kenya. Parasit Vectors. 2011:4:122.

26. Adano WR, Dietz T, Witsenburg K, Zaal F. Climate change, violent conflict and local institutions in Kenya's drylands. J Peace Res. 2012:49:65-80.

27. Agnew ADQ, Mwendia CM, Oloo GO, Roderick S, Stevenson P. Landscape monitoring of semi-arid rangelands in the Kenyan Rift Valley. Afr J Ecol. 2000;38:277-85

28. Lin S, DeVisser MH, Messina JP. An agent-based model to simulate tsetse fly distribution and control techniques: a case study in Nguruman, Kenya. Ecol Model. 2015;314:80-9.

29. Osano PM. Said MY, deLeeuw J, Moiko SS, OleKaelo D, Schomers S, et al. Pastoralism and ecosystem-based adaptation in Kenyan Masailand. Int J Clim Chang Strateg Manag. 2013:5:198-214

30. Allsopp R, Baldry DAA. general description of the Lambwe Valley area of South Nyanza District, Kenya. Bull World Heal. Organ. 1972;47:691-7.

31. Tarimo-Nesbitt RA, Golder TK, Chaudhury MF. Trypanosome infection rate in cattle at Nguruman, Kenya. Vet Parasitol. 1999:81:107-17.

32. Abila PP, Slotman MA, Parmakelis A, Dion KB, Robinson AS, Muwanika VB, et al. High levels of genetic differentiation between Ugandan Glossina fuscipes fuscipes populations separated by Lake Kyoga. PLoS Negl Trop Dis. 2008; $\mathrm{e} 242: 2$.

33. Hyseni C, Beadell JS, Ocampo-Gomez J, Okedi LM, Gaunt M, Caccone A. The G. m. morsitans (Diptera: Glossinidae) genome as a source of microsatellite markers for other tsetse fly (Glossina) species. Mol Ecol Resour. 2011;11:586-9.

34. Ouma JO, Cummings MA, Jones KC, Krafsur ES. Characterization of microsatellite markers in the tsetse fly, Glossina pallidipes (Diptera: Glossinidae). Mol Ecol Notes. 2003;3:450-3.

35. Baker MD, Krafsur ES. Identification and properties of microsatellite markers in tsetse flies Glossina morsitans sensu lato (Diptera: Glossinidae). Mol Ecol Notes. 2001:1:234-6.

36. Agata K, Alasaad S, Almeida-Val VMF, Álvarez-Dios JA, Barbisan F, Beadell JS, et al. Permanent genetic resources added to molecular ecology resources database 1 December 2010-31 January 2011. Mol Ecol Resour. 2011;11:586-9.

37. Van Oosterhout C, Hutchinson WF, Wills DPM, Shipley P. MICRO-CHECKER Software for identifying and correcting genotyping errors in microsatellite data. Mol Ecol Notes. 2004:4:535-8.

38. Rousset F. genepop'007: a complete re-implementation of the genepop software for Windows and Linux. Mol Ecol Resour. 2008:8:103-6.

39. Benjamini Y, Hochberg Y. Controlling the false discovery rate: a practical and powerful approach to multiple testing. J R Stat Soc B. 1995;57:289-300.

40. Peakall R, Smouse PE. GenAlEx 6.5: genetic analysis in Excel. Population genetic software for teaching and research - an update. Bioinformatics. 2012;28:2537-9.

41. Goudet J. FSTAT, a program to estimate and test gene diversities and fixation indices (version 29.3). 2001.

42. Excoffier $L$, Lischer HE. Arlequin suite ver 3.5: a new series of programs to perform population genetics analyses under Linux and Windows. Mol Ecol Resour. 2010;10:564-7.

43. Waples RS. A generalized approach for estimating effective population size from temporal changes in allele frequency. Genetics. 1989;121:379-91.

44. Jorde PE, Ryman N. Unbiased estimator for genetic drift and effective population size. Genetics. 2007;177:927-35. 
45. Do C, Waples RS, Peel D, Macbeth GM, Tillett BJ, Ovenden JR. NeEstimator V2: re-implementation of software for the estimation of contemporary effective population size ( $\mathrm{Ne}$ ) from genetic data. Mol Ecol Resour. 2014;14: 209-14.

46. Pollak EA. A new method for estimating the effective population size from allele frequency changes. Genetics. 1983;104:531-48.

47. Piry S, Luikart G, Cournet JMB. A computer program for detecting recent reductions in the effective population size using allele frequency data. J Hered. 1999;90:502-3.

48. Luikart G, Allendorf FW, Cornuet JM, Sherwin WB. Distortion of allele frequency distributions provides a test for recent population bottlenecks. J Hered. 1998;89:238-47

49. Evanno G, Regnaut S, Goudet J. Detecting the number of clusters of individuals using the software STRUCTURE: a simulation study. Mol Ecol. 2005:14:2611-20.

50. Kopelman NM, Mayzel J, Jakobsson M, Rosenberg NA, Mayrose I. Clumpak: a program for identifying clustering modes and packaging population structure inferences across K. Mol Ecol Resour. 2015;15:1179-91.

51. Earl D, vonHoldt BM. STRUCTURE HARVESTER: a website and a program for vizualizing STRUCTURE output nad implementing the Evanno method. Conserv Genet Resour. 2012:4:359-61.

52. Saitou N, Nei M. The neighbor-joining method: a new method for reconstructing phylogenetic trees. Molec Biol Evol. 1987;4:406-25.

53. Nei M, Tajima F, Tateno Y. Accuracy of estimated phylogenetic trees from molecular data - I. Distantly related species. J Mol Evol. 1983;18:387-404.

54. Kamvar ZN, Brooks JC, Grünwald NJ, Novel R. tools for analysis of genomewide population genetic data with emphasis on clonality. Front Genet. 2015;6:1-10

55. Kamvar ZN, Tabima JF, Grünwald NJ. Poppr: an R package for genetic analysis of populations with clonal, partially clonal, and/or sexual reproduction. PeerJ. 2014;2:e281.

56. Wright S. The genetical structure of populations. Ann Hum Genet. 1949;15: 323-54

57. Weir BS, Cockerham CC. Estimating F-Statistics for the analysis of population structure. Evolution. 1984;38:1358-70.

58. Goudet J, Raymond M, De Meeüs T, Rousset F. Testing differentiation in diploid populations. Genetics. 1996;144:1933-40.

59. Jensen JL, Bohonak AJ, Kelley ST. Isolation by distance, web service. BMC Genet. 2005;6:13.

60. Ersts PJ. Geographic distance matrix generator (version 1.2. 3 American Museum of Natural History, Center for Biodiversity and Conservation. Available from http://biodiversityinformatics.amnh.org/open_source/gdmg.

61. Mantel N. The detection of disease clustering and a generalize regression approach. Cancer Res. 1967;27:209-20.

62. Abd-Alla AMM, Adun H, Parker AG, Vreysen MJB, Bergoin M. The antiviral drug valacyclovir successfully suppresses salivary gland hypertrophy virus (SGHV) in laboratory colonies of Glossina pallidipes. PLoS One. 2012;7:e38417.

63. Boucias DG, Kariithi HM, Bourtzis K, Schneider DI, Kelley K, Miller WJ, et al. Transgenerational transmission of the Glossina pallidipes hytrosavirus depends on the presence of a functional symbiome. PLoS One. 2013;8: e61150.

64. Olet PA, Opiyo E, Robinson AS. Sexual receptivity and age in Glossina pallidipes Austen (Dipt., Glossinidae). J Appl Entomol. 2002;126:86-91.

65. Fisher RA. On the interpretation of $X^{2}$ from contingency tables, and the calculation of P. J R Stat Soc. 1922:85:87-94.

66. Jackson CHN. The biology of tsetse flies. Biol Rev. 1949;24:174-99

67. Brightwell R, Dransfield DR, Kyorku C. Development of low-cost traps and odour baits for Glossina pallidipes and G. Iongipennis in Kenya. Med Vet Entomol. 1991;5:153-64.

68. Dyer NA, Furtado A, Cano J, Ferreira F, Odete Afonso M, Ndong-Mabale N, et al. Evidence for a discrete evolutionary lineage within Equatorial Guinea suggests that the tsetse fly Glossina palpalis palpalis exists as a species complex. Mol Ecol. 2009;18:3268-82.

69. Beadell JS, Hyseni C, Abila PP, Azabo R, Enyaru JCK, Ouma JO, et al. Phylogeography and population structure of Glossina fuscipes fuscipes in Uganda: Implications for control of tsetse. PLoS Negl Trop Dis. 2010;e636:4.

70. Hyseni C, Kato AB, Okedi LM, Masembe C, Ouma JO, Aksoy S, et al. The population structure of Glossina fuscipes fuscipes in the Lake Victoria basin in Uganda: implications for vector control. Parasit Vectors. 2012;5:222.

71. Echodu R, Sistrom M, Hyseni C, Enyaru J, Okedi L, Aksoy S, et al. Genetically distinct Glossina fuscipes fuscipes populations in the Lake Kyoga region of
Uganda and its relevance for human African trypanosomiasis. Biomed Res Int. 2013:614721.

72. Echodu R, Beadell JS, Okedi LM, Hyseni C, Aksoy S, Caccone A. Temporal stability of Glossina fuscipes fuscipes populations in Uganda. Parasit Vectors. 2011:4:19.

73. Opiro R, Saarman NP, Echodu R, Opiyo EA, Dion K, Halyard A, et al. Evidence of temporal stability in allelic and mitochondrial haplotype diversity in populations of Glossina fuscipes fuscipes (Diptera: Glossinidae) in northern Uganda. Parasit Vectors. 2016;9:258.

74. Beaumont MA, Zhang W, Balding DJ. Approximate Bayesian computation in population genetics. Genetics. 2002;162:2025-35.

75. Schwartz MK, Tallmon DA, Luikart G. Review of DNA-based census and effective population size estimators. Anim Conserv. 1998:1:293-9.

76. Waples RS, Do CLDNE. A program for estimating effective population size from data on linkage disequilibrium. Mol Ecol Resour. 2008:8:753-6.

77. Muya E, Sijali I, Radiro M, Okoth P. Comparative analysis of water saving techniques for irrigating more land with less water in Nguruman Scheme, Kenya: Design principles and practices. Irrig Drain. Syst Eng. 2016;5:158.

78. Bekure S, deLeeuw PN, Grandin BE. Neate, PJH. Maasai hearding: An analysis of the livestock production system of Maasai; 1991.

79. Spear SF, Balkenhol N, Mcrae B, Scribner KT, Scribner KIM, Spear SF, et al. Use of resistance surfaces for landscape genetic studies. Use of resistance surfaces for landscape genetic studies: considerations for parameterization and analysis. Mol Ecol. 2010;19:3576-91.

80. McRae B. Isolation by resistance. Evolution. 2006;60:1551-61.

81. Wang I, Bradburd G. Isolation by environment. Mol Ecol. 2014;23:5649-62.

82. Bradburd GS, Ralph PL, Coop GM. Disentangling the effects of geographic and ecological isolation on genetic differentiation. Evolution. 2013;67:3258-73.

\section{Submit your next manuscript to BioMed Central and we will help you at every step:}

- We accept pre-submission inquiries

- Our selector tool helps you to find the most relevant journal

- We provide round the clock customer support

- Convenient online submission

- Thorough peer review

- Inclusion in PubMed and all major indexing services

- Maximum visibility for your research

Submit your manuscript at www.biomedcentral.com/submit
) Biomed Central 